\title{
Editorial
}

\section{MIMO Transmission with Limited Feedback}

\author{
Markus Rupp,, ${ }^{1}$ Ana I. Pérez-Neira, ${ }^{2}$ David Gesbert, ${ }^{3}$ and Christoph F. Mecklenbräuker ${ }^{1}$ \\ ${ }^{1}$ Institute of Communications and Radio-Frequency Engineering, Vienna University of Technology, Gusshausstrasse 25/389, \\ 1040 Vienna, Austria \\ ${ }^{2}$ Department of Signal Theory and Communications, Technical University of Catalonia, North Campus, Jordi Girona 1-3, \\ 08034 Barcelona, Spain \\ ${ }^{3}$ Eurecom Institute, 2229 Route des Cretes, BP 193, 06904 Sophia Antipolis Cedex, France
}

Correspondence should be addressed to Markus Rupp, mrupp@nt.tuwien.ac.at

Received 20 February 2008; Accepted 20 February 2008

Copyright (c) 2008 Markus Rupp et al. This is an open access article distributed under the Creative Commons Attribution License, which permits unrestricted use, distribution, and reproduction in any medium, provided the original work is properly cited.

During the past decade, multiple-antenna transmission (MIMO) systems have matured. However, when comparing their potential capacities with their achieved throughputs, we notice large gaps. The price for the MIMO advantages is increased cost for the transceiver hardware and antenna subsystems, combined with high algorithmic complexity in baseband processing and protocols. For instance, water filling and power loading do not seem feasible due to the large amount of required channel state information. State-of-the-art systems with MIMO enhancements like 3G cellular networks, WiMax, and WLAN support very limited feedback only. Nevertheless, adaptive modulation and coding (AMC) schemes, selective space-time coding, as well as antenna selection have shown that significant improvements are achievable even with very limited feedback. In this setting, MIMO-OFDM schemes are of central interest to industry and academia. For instance, an important challenge is to find an adequate representation of the MIMO channel's quality-independently of the system architecture and signal processing techniques currently available. A proper labeling or characterization of the MIMO channel quality regardless of the spatial processing to be used enables deciding on the reception or transmission strategy to use (e.g., with or without channel state information, to optimize diversity or rate, etc.) and, thus, on the amount of feedback that is required in transmission. MIMO transmission can be pointto-point or distributed; in fact, when looking not just into the physical layer, but also into the link layer, feedback load is especially critical in multiuser MIMO systems because of its much higher number of degrees of freedom. Opportunistic scheduling strategies have been developed, which (more or less heuristically) take into account the requirements on QoS.
This special issue focuses on such transmission systems with limited feedback and provides an overview of the state of the art. We received 23 submissions, out of which we finally selected eleven for this special issue.

The paper entitled "Antenna subset selection for cyclic prefix-assisted MIMO wireless communications over frequency selective channels" by A. Wilzeck and T. Kaiser is a tutorial paper on antenna selection techniques in broadband transmissions. In broadband MIMO systems aiming to provide high data rate links, the employed signal bandwidth is typically larger than the coherence bandwidth of the channel, so that the channel will be of frequency selective nature. While most contributions in literature consider only the frequency flat case, this paper shows how MIMO orthogonal frequency division multiplexing systems and MIMO single-carrier systems can be deployed by simple low complex equalization techniques.

The contribution entitled "A design framework for scalar feedback in MIMO broadcast channels" by R. Francisco and D. Slock reports on joint linear beamforming and scheduling techniques. The feedback consists of channel direction information (CDI) based on a predetermined codebook and a scalar metric with channel quality information (CQI) to perform user scheduling. An approximation on the sum rate is provided for the proposed family of metrics, which is validated through simulations and provides a means of simple comparison between transmission schemes and scalar feedback techniques. Particularly, the sum rates of SDMA and time-division multiple access (TDMA) are compared in the extreme regimes: large number of users, high SNR, and low SNR.

In the paper which is entitled "Feedback reduction in uplink MIMO OFDM systems by chunk optimization” by 
E. Jorswieck et al., the authors propose to maximize the weighted sum rate of a MIMO OFDM MAC under individual power constraints and chunk size constraints. An efficient iterative algorithm is developed and convergence is proved.

Cooperative antenna systems have recently become a hot research topic, as they promise significantly higher spectral efficiency than conventional cellular systems. In the paper entitled "Efficient feedback via subspace-based channel quantization for distributed channel quantization for distributed cooperative antenna systems with temporally correlated channels" by J. H. Kim et al., the authors study how to provide base stations with downlink channel information for transmit filtering and they propose a novel feedback scheme via a subspace-based channel quantization method. Their scheme adopts the chordal distance as a channel quantizer criterion and performs as well as the permanent full feedback scheme with a much smaller amount of uplink resources.

The contribution entitled "Experimental evaluation of adaptive modulation and coding in MIMO WiMAX with limited feedback" by C. Mehlführer et al., evaluates the throughput performance of the IEEE802.16-2004 transmission system with adaptive modulation and coding by outdoor measurements. The paper shows that in the reported scenarios, the measured throughput is far from being achievable; the loss is mainly caused by a too simple convolutional coding as well as poor channel estimation.

In their paper entitled "Feedback quantization for linear precoded spatial multiplexing" C. Simon and G. Leus propose a comparative analysis of recently developed quantization schemes for use in linear precoded spatially multiplexed MIMO systems. They consider various forms of distortion functions leading to vector quantization codebooks. The correlation properties of radio channel are made use of in order to derive novel feedback compression schemes.

B. Mondal and R. Heath consider in the paper entitled "A diversity guarantee and SNR performance for unitary limited feedback MIMO systems" the problem of MIMO channel quantizing and the effect of limited feedback on the MIMO diversity performance. Their analysis indicates that there exists a threshold for feedback limitation above which no loss of diversity is incurred from the quantizing. The threshold is determined as a function on system parameters like number of antennas, SNR, and so forth.

The paper by K.-K. Wong and J. Chen, entitled "Timedivision multiuser MIMO with statistical feedback" investigates a time-division MIMO downlink system where users are given individual outage rate probability constraints. Assuming that the transmitter knows only the statistical information about the channel, the authors develop a scheme optimizing power allocation and time-sharing parameters. They modify the initial nonconvex problem into a suboptimal convex optimization problem which performs nearly as well as the global optimum.

The paper entitled "Stable transmission in the timevarying MIMO broadcast channel” by A. L. Anderson et al. studies the degradation caused by using outdated channel state information at the transmitter to construct a signaling scheme for the current channel. They use both traditional channel models and measured channel realizations for analysis. With measured data from an outdoor urban environment, they show that stable subspaces exist upon which transmission is possible without any instantaneous channel state information at the transmitter.

K. Huang et al. focus on uplink space division multiple access with limited feedback. Their paper is entitled "Uplink SDMA with limited feedback: throughput scaling." They analyze the scaling of throughput with respect to the numbers of users, antennas, and feedback bits and derive design guidelines.

M. Kountouris et al. consider the problem of feedback reduction in a multiuser multiple-antenna downlink system with more users than transmit antennas, under partial channel knowledge at the transmitter. In their paper entitled "Scheduling for multiuser MIMO downlink channels with ranking-based feedback," it is shown that ranking-based user selection substantially reduces the required feedback rate with negligible decrease in multiuser diversity gain and throughput when users have i.i.d. channels.

\author{
Markus Rupp \\ Ana I. Pérez-Neira \\ David Gesbert \\ Christoph F. Mecklenbräuker
}

